\title{
Effect of Artificial Light on the Growth of Plants.
}

THE effect of light on plant life has been the subject of many scientific investigations. So long ago as I 880 Siemens showed that continuous exposure to arc lamps promoted luxuriance of foliage, greater intensity of colouring, and more rapid ripening of fruit. It is common knowledge that plants grown in partial shade are apt to be weakly, and that the flowers, if produced, have in general a fainter shade of colour. Other examples were mentioned by Schübeler in a communication to the British Association in $188 \mathrm{I}$. The extraordinary rapid growth of vegetation in the Arctic regions during the period of uninterrupted daylight has been the subject of comment; likewise the rapid ripening of corn in reyions of Norway and Sweden where the summer does not exceed two months, but where, during this period, the sun scarcely sets.

It therefore seems somewhat surprising that greater use has not hitherto been made of the use of artificial light in horticulture. There have, in the past, been intermittent experiments, but results have been somewhat discouraging, and the probable reason is not far to seek. It is only within recent years, when convenient methods of measuring light have become general, that the great disparity between the intensity of full daylight and artificial light has come to be properly understood. The illumination derived from direct sunlight in summer may attain ro, ooo footcandles, and even with a cloudy sky a value of several thousand foot-candles is quite usual. On the other hand, the average illumination in an artificially lighted building is frequently less than three footcandles; a general illumination of ten foot-candles would be considered exceptionally bright. The marvellous power of adaptation of the eye enables it to utilise these extreme variations in illumination. But it can be readily appreciated that unless an artificial illumination of at least several hundreds of foot-candles can be provided, the effect on the growth of plants would not be marked.

Until a few years ago there were few sources of light that could be conveniently applied to produce such high intensities, and the cost was commercially prohibitive. But the advent of such sources as the electric gas-filled lamp, furnishing tooo c.p. or more, and capable of being equipped with suitable reflectors to concentrate the available light within a narrow area, has improved the position considerably. For some time experiments have been in progress at various agricultural experimental stations in the United States, and some results of these researches were recently summarised in a series of papers read before the American Jlluminating Engineering Society.

The light was provided by electric gas-filled lamps, and values of illumination varying from 300 to 1500 foot-candles were furnished. The exposure to artificial light was supplementary to the natural lighting and had a material effect in hastening the growth of seedlings. Provided that proper attention was paid to the conditions which are ordinarily observed in horticulture--for example, the provision of sufficient space for normal development of each plant, proper soil, maintenance of correct conditions in regard to moisture and temperature, etc.-the more rapid growth was not attended by weakness. On the contrary, the plants were exceptionally robust and, it is stated, less liable to the ravages of fungus and other diseases.

The most striking effect of the increased light, however, was in hastening the time of blooming. On the average, flowering plants came into bloom eight days earlier than when no artificial light was used.
Mr. R. B. Harvey, recording tests made at the Minnesota Experiment Station, mentions that the effect of continuous illumination is to cause the accumulation of large quantities of starch in the plants; thus, buckwheat was so full of starch that the grains may be pulverised in the hand. Apparently the desirable illumination varies for different plants. Strawberries, for example, did not bloom at 500 footcandles, but set fruits at intensities of more than I $_{5} \mathrm{OO}$ foot-candles. Prof. H. Findlay, of Columbia University, reports similar experience. The development of bloom in most plants was accelerated by about eight days; marguerites came into bloom eighteen days after the lights were turned on, when similar plants, grown only under sunlight, were scarcely showing bud.

Another question, apart from effect of intensity, is the influence of colour of light. It would seem that light from the electric gas-filled lamp may, as regards its effect on vegetation, be regarded as broadly similar to daylight, and probably the same would apply to other illuminants giving continuous spectra and approximately white light. Early experiments on the effect of colour seem to have been somewhat inconclusive, owing to uncertainty regarding the relative intensity. Thus, the effect of inserting a pane of blue glass is naturally to diminish the brightness of the light very considerably, besides restricting the rays to the blue end of the spectrum. Some remarkable results have, however, been recorded by M. Camille Flammarion, ${ }^{1}$ who attempted the culture of the sensitive mimosa plant under light of different colours. Plants placed in blue light scarcely gained in growth at all, and also appeared to be in a comatose state, showing none of the sensibility to touch characteristic of the mimosa. Plants grown under red light were, on the other hand, four times as big as those grown under white light, developed well-marked flower balls, and were in an extremely sensitive state. From these and other experiments the conclusion has been drawn that red, orange, and yellow rays, by building up complex organic chains of molecules, stimulate plant life, wherreas blue rays have an arresting effect. Ultra-violet rays, when present in excess, undoubtedly may have a prejudicial effect, causing shrivelling of the leaves and ultimately the death of the plant. In judicious proportions, they may, however, prove beneficial, as is believed to be the case in regard to the human body. Instances of their use to destroy greenfly and similar pests have been recorded.

Here, therefore, is a good field for experiment. We have still much to learn regarding the effects of sustained exposure to strong white light, whilst the effect of light of specific wave-lengths has been only very imperfectly studied. It would be very desirable for corroborative investigations, similar to those conducted at research stations in the United States, to be undertaken by leading horticulturists in Great Britain. At present it is not anticipated that artificial light could be economically applied to the production of common foodstuffs on a large scale; but it is believed that its use may prove extremely valuable for the purpose of raising scarce fruits and flowers out of season. It is even possible-though apparently this requires confirmation-that the work might be conducted entirely by artificial light in a well-heated and ventilated cellar in cases where no garden is available, thus bringing this field of investigation within the scope of the town-dweller in congested districts.

1 Bulletin de la Société Astronomique de France, Aug. 1897.

$$
\text { NO. } 2872 \text {, VOL. I } 14 \text { ] }
$$

